\title{
Significados sobre las actitudes hacia las matemáticas por estudiantes universitarios multiculturales
}

\author{
Attitudes meanings towards mathematics by multicultural college students
}

William Oswaldo Flores López ${ }^{1}$

\begin{abstract}
Resumen
Esta investigación ha analizado los significados sobre las actitudes hacia las matemáticas por estudiantes universitarios en contextos multiculturales. Es un estudio cualitativo con un abordaje desde la fenomenología hermenéutica con la participaron de 40 estudiantes universitarios en grupos de discusión. El procesamiento y la interpretación de la información fue a través del análisis del contenido manifiesto (literal) y latente (discurso). Los resultados revelan que las actitudes son cualidades de hombres y mujeres que se manifiestan como actuaciones, conductas, sentimientos, valores, capacidades y comportamientos hacia un constructo u objeto mediante el estímulo a una reacción o una petición en la resolución de problemas en contextos multiculturales. Se concluye que existen significados propios y compartidos en relación con las actitudes y los factores actitudinales hacia las matemáticas.
\end{abstract}

Palabras clave: actitudes; matemáticas; factores actitudinales; significados propios; significados compartidos.

\section{Abstract}

This research has analyzed the meanings of attitudes towards mathematics by university students in multicultural contexts. It is a qualitative study with an approach from hermeneutic phenomenology with the participation of 40 university students in discussion groups. The processing and interpretation of the information was through the analysis of the manifest (literal) and latent (discourse) content. The results reveal that attitudes are qualities of men and women that are manifested as actions, behaviors, feelings, values, capacities and behaviors towards a construct or object by stimulating a reaction or a request in solving problems in multicultural contexts. It is concluded that there are proper and shared meanings in relation to attitudes and attitudinal factors towards mathematics.

Keywords: Attitudes; maths; attitudinal factors; proper meanings; Shared meanings.

\section{Introducción}

Las actitudes hacia las matemáticas son valoraciones o sentimientos de aceptación o rechazo hacia un constructo u objeto que mujeres y hombres manifiestan en un contexto de resolución de problemas con emociones y creencias (Flores \& Auzmendi, 2018; y Flores, 2019). El estudio de las actitudes hacia las matemáticas es fundamental porque impide una progresión en el aprendizaje de las matemáticas. En la literatura, existen estudios que tratan de identificar las actitudes hacia las matemáticas. Por ejemplo,

\footnotetext{
1 Doctor en Educación. Profesor Investigador de la Universidad de las Regiones Autónomas de la Costa Caribe Nicaragüense. Email: william.flores@uraccan.edu. ni ORCID: https://orcid.org/0000-0002-1016-1620
}

Recibido: 23/04/2020 - Aprobado: 28/05/2020 
Cardoso et al. (2012), dicen que las actitudes configuran una información valiosa tanto para la planeación e implementación de los procesos de enseñanza por parte de los docentes, como para el desarrollo de los procesos formativos a generar en el estudiantado quienes van a seguir interactuando con dicha disciplina no sólo en el ámbito académico, sino en el laboral.

Desde esta perspectiva, el propósito de esta investigación es analizar los significados propios y compartidos sobre las actitudes hacia las matemáticas por estudiantes universitarios en contextos multiculturales. A partir de lo anterior, se planteó la técnica de grupos de discusión porque es una técnica de investigación cualitativa que, trabaja con el habla. Se utiliza la técnica de análisis de contenido manifiesto (literal) y latente (discurso) expuesto por Babbie (1974).

\section{Revisión de literatura}

En la educación en general y particularmente en la educación matemática, las actitudes pueden considerarse uno de los aspectos psicológicos que han alcanzado más difusión. Este hecho puede asociarse a la calidad educativa de las matemáticas. Por ello, es importante, en primera instancia, caracterizar el término de actitudes. Para ello, partimos de la definición de Allport (1935), las actitudes son un estado mental y neurofisiológico de disponibilidad, organizado por la experiencia que ejerce una influencia directiva sobre las reacciones del individuo hacia todos los objetos y a todas las situaciones que se relacionan con ella. Por su parte, Rokeach (1968), opinó que las actitudes son organizaciones de creencias relativamente permanentes que, predisponen a responder de un modo preferencial ante un objeto o situación. En cambio, Likert (1976) afirmó que, las actitudes son disposiciones hacia una acción manifiesta.

En la línea de las ideas anteriores, Auzmendi (1992) propone factores actitudinales que, miden el nivel de ansiedad, agrado, confianza, utilidad y motivación hacia las matemáticas. El agrado es un factor que hace referencia al aspecto de agrado o disfrute que provoca el trabajo matemático; el factor ansiedad se refiere al sentimiento de temor que el estudiante manifiesta ante las matemáticas; el factor de motivación puede interpretarse como la motivación que siente el estudiante hacia el estudio y utilización de las matemáticas; el factor utilidad hace referencia al valor que el estudiante otorga a las matemáticas, a la utilidad que él percibe que puede tener esta materia para su vida futura profesional; y el factor confianza puede interpretarse como el sentimiento de confianza que provoca la habilidad en matemáticas.

\section{Materiales y métodos}

La investigación en el ámbito educativo exige un proceso organizado y sistemático para comprender, conocer y explicar la realidad educativa, por ello, esta investigación es de carácter cualitativa porque permite describir la realidad educativa con el fin de llegar a la comprensión o a la transformación de dicha realidad, a partir del significado atribuido por las personas que la integran (Bisquerra, 2012). Desde esta perspectiva, el abordaje de esta investigación es desde la fenomenología hermenéutica (Heidegger, 1982; Gadamer, 2000; y Ricoeur, 2000), la fenomenología ${ }^{2}$ se encarga precisamente de desvelar lo que hay oculto en la consciencia, aquello que se construye en las experiencias y que poco a poco van construyendo significados acerca del mundo y de todo aquello inmerso en el espacio vital. Por tanto, se podría acceder al fenómeno de las actitudes hacia las matemáticas como experiencia de vida y desarrollo humano desde el contexto natural, así, toda acción del ser humano, todo saber, toda experiencia no tiene sentido más que en la medida en que se pueda hablar y escribir de ella.

A partir de lo anterior, se planteó la técnica de grupos de discusión ya que, es una técnica de investigación cualitativa que trabaja con el habla. Entonces, para la configuración de los grupos se combinó el criterio de homogeneidad y heterogeneidad sugerido por Krueger (1991), que manifiesta que cada grupo debe ser lo suficiente homogéneo para evitar situaciones de inhibición, condicionamiento o prevalencia de

\footnotetext{
2 La investigación fenomenológica es el estudio de la experiencia vital, del mundo de la vida, de la cotidianidad. Lo cotidiano, en sentido fenomenológico, es la experiencia pre-reflexiva, la experiencia no conceptualizada o categorizada (...). La investigación fenomenológica es la descripción de los significados vividos, existenciales (Melich, 1994).
} 
opiniones y a la vez la heterogeneidad en relación con el mayor número de variables diferenciadoras dentro de un mismo colectivo. Además, se garantizó que existiera la posibilidad de que los sujetos tuvieran opción de intervenir durante un espacio razonable de tiempo para exteriorizar sus respectivas opiniones; se decidió que, los grupos estuvieran formados por un mínimo de 3 personas y un máximo de 6. A la vez, se combinó el género, la edad y carrera. En definitiva, aplicando los criterios descritos, quedaron configurados seis grupos de discusión para un total de 40 personas (Mujeres=20; Hombres=20). En este sentido, la discusión fue guiada por palabras claves como: actitud, ansiedad, utilidad, agrado, motivación y confianza hacia las matemáticas.

Para el análisis y procesamiento de la información, la técnica utilizada fue el análisis de contenido manifiesto (literal) y latente (discurso) expuesto por Babbie (1974). El análisis de contenido manifiesto es una técnica que da cuenta cómo se transmiten los significados y estudia los contenidos literales de las narrativas individuales o grupales. El análisis de contenido latente (discurso), por su parte, tiene que ver con el habla y el texto producido (Wethrell \& Potter, 1996). El análisis de discurso de esta investigación integró elementos de análisis crítico del discurso de Wodak y Meyer (2009). Este acercamiento ve el lenguaje como una práctica social y considera crucial el análisis del contexto en el que se desarrolla. Según Wodak et al. (2009), el análisis crítico del discurso busca conocer el desarrollo de los significados y cómo se producen.

\section{Resultados y discusión}

La actitud hacia las matemáticas es un constructo que juega un papel importante en los trayectos curriculares de la formación de profesionales. En Gómez-Chacón (2000), se dice que las actitudes son un campo de creencias, sentimientos y estado de ánimo que transcienden el dominio de la cognición. Mientras que Guerrero et al. (2002), las conceptualizan como una predisposición conformada de acuerdo con una serie de convicciones y sentimientos que hacen que el sujeto reaccione con sus creencias y sentimientos. De acuerdo con estos conceptos, se puede pensar en una definición propia de actitudes:

Las actitudes son cualidades de hombres y mujeres que se manifiestan como actuaciones, conductas, sentimientos, valores, capacidades y comportamientos hacia un constructo u objeto mediante el estímulo a una reacción o una petición en la resolución de problemas en contextos multiculturales.

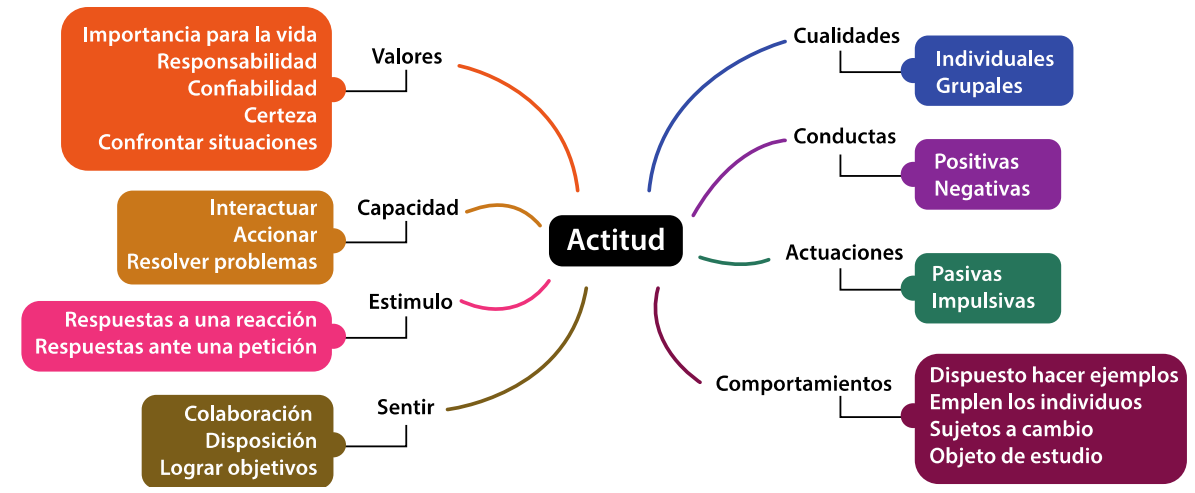

Figura 1: Concepción de actitud hacia las matemáticas.

Por otra parte, según Gresham (2010), la ansiedad hacia las matemáticas es entendida como un miedo irracional hacia esta disciplina que, dificulta la realización de cálculos numéricos y la resolución de problemas de matemáticas en diversas situaciones de la vida académica y cotidiana del sujeto, como la impotencia y el pánico que experimenta el sujeto cuando se le plantea alguna tarea de matemáticas (Bursal \& Paznokas, 2006), causa de una experiencia negativa o humillante con las matemáticas por falta de conocimiento o la aplicación inadecuada de los conceptos matemáticos (Furner \& Berman, 2003). Desde esta perspectiva, se propone el concepto siguiente: "la ansiedad son comportamientos de inseguridad, desconfianza y preocupación de hombres y mujeres que se exteriorizan como conductas, sentimientos y capacidades en el momento de sentir y pensar la solución de un problema en contexto multiculturales". 


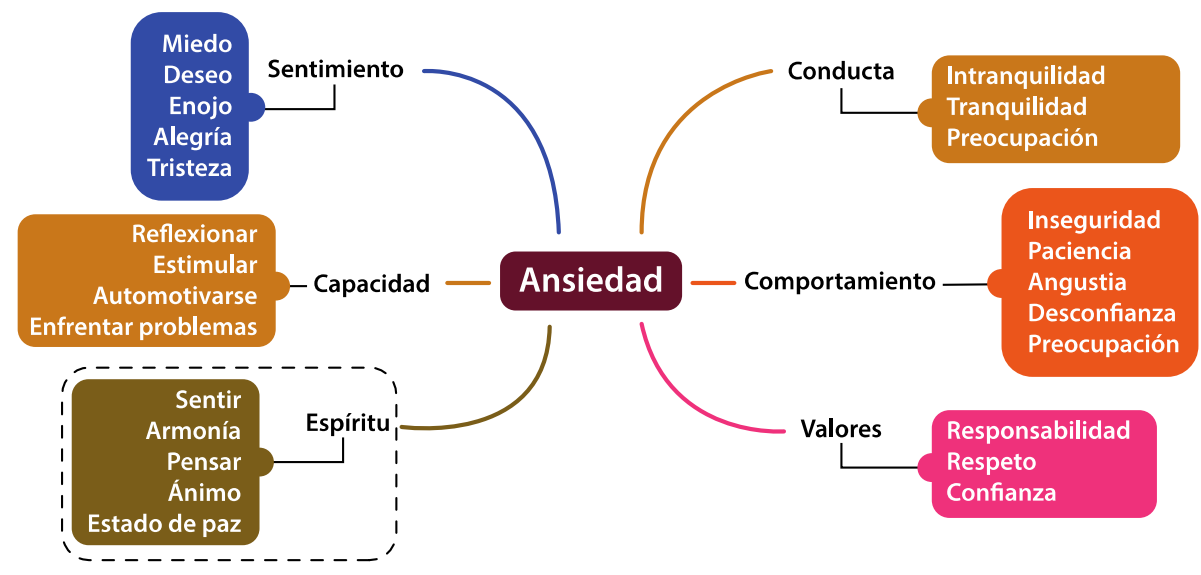

Figura 2: Concepción de ansiedad hacia las matemáticas.

En el caso de la motivación es el conjunto de razones por las que las personas se comportan de la forma en que lo hacen. Dichos comportamientos se caracterizan por ser vigoroso, dirigido y sostenido (Santrock, 2001). Es por ello, que existen dos tipos de motivación: intrínseca y extrínseca. La motivación intrínseca favorece el desarrollo de grupos informales al margen de las estructuras formales, lo cual permite la rápida resolución de problemas, la transferencia de las mejores prácticas y el desarrollo de habilidades profesionales al compartir experiencias y conocimiento tácito (Wenger y Snyder, 2000). Mientras que en la motivación extrínseca el aprendizaje es secundario, no es permanente y no se puede garantizar; es el medio para conseguir otros fines. Por consiguiente, se propone la definición siguiente: "la motivación es el conjunto de habilidades, capacidades y comportamientos que hombres y mujeres interiorizan de forma interna y externa para auto estimularse su estado de ánimo y sentimientos de cara a la resolución de problemas en contextos multiculturales".

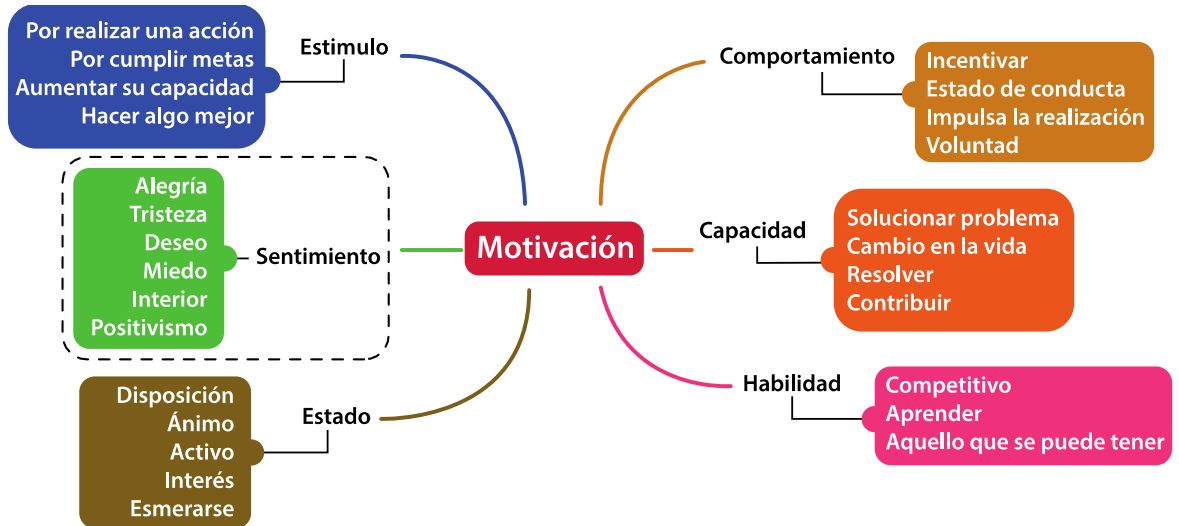

Figura 3: Concepción de motivación hacia las matemáticas.

En relación con el agrado, se puede definir como el disfrute que percibe el estudiante en el trabajo con la matemática y su estudio (Palacios et al., 2014); así mismo, hace referencia al disfrute que provoca el trabajo matemático (Auzmendi, 1992). A continuación, se propone una definición de agrado: "el agrado es un sentimiento de disfrute, felicidad y comportamientos que hombres y mujeres manifiestan al momento de usar su capacidad de resolver problemas en contextos multiculturales". 


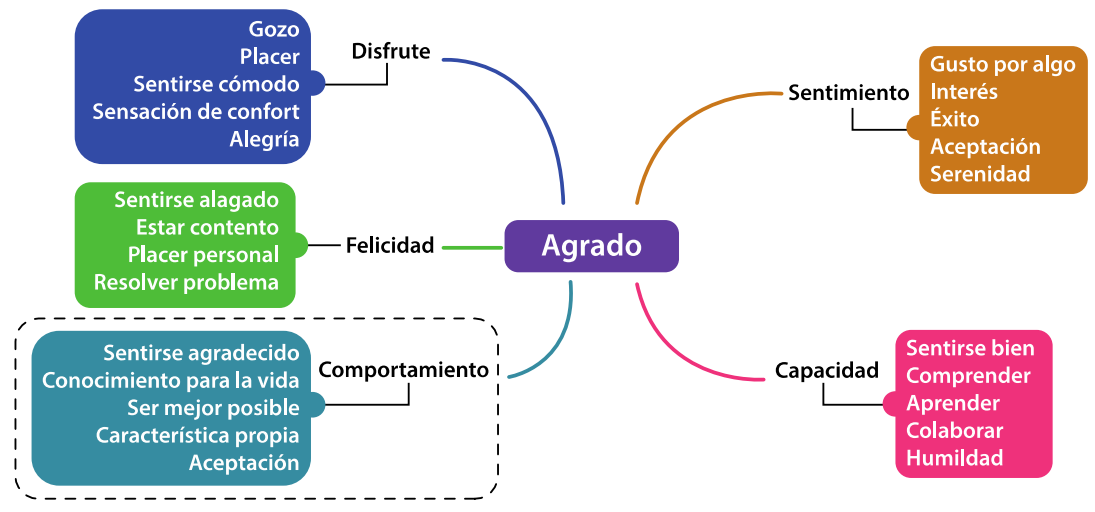

Figura 4: Concepción de agrado hacia las matemáticas.

El concepto de confianza es un constructo relevante de analizar, se puede definir como un sentimiento que provoca la habilidad y realización de la práctica matemática (Flores \& Auzmendi, 2015). La confianza se refiere a la satisfacción que siente el estudiante hacia el estudio de la materia, la confianza que tiene en sí mismo y el valor que otorga a la materia de cara a su futuro profesional (Mato \& De la Torre, 2010). En este sentido, se define el concepto de confianza: "la confianza es un sentimiento de éxito y bienestar que provoca en hombres y mujeres el valor y la capacidad de construir ideas para resolver problemas a partir del reconocimiento y la familiarización del contexto multicultural".

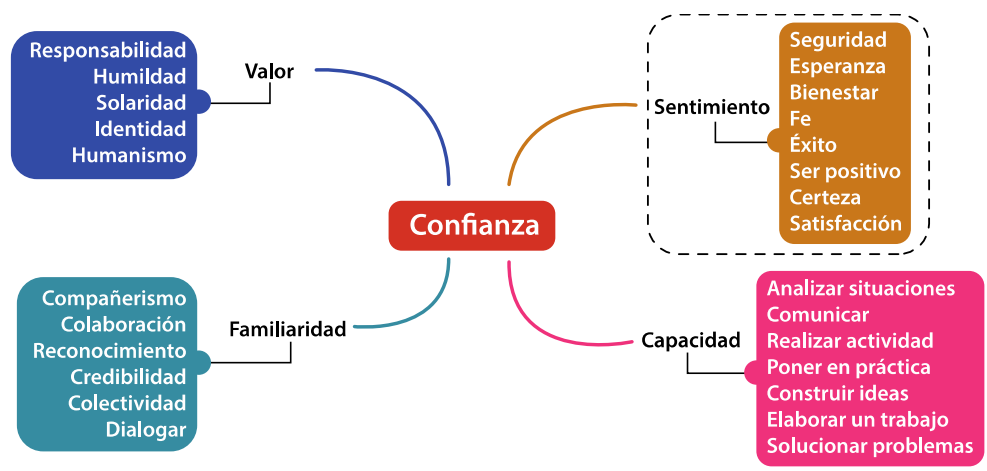

Figura 5: Concepción de confianza hacia las matemáticas.

La percepción de utilidad hacia las matemáticas es uno de los principales componentes para el estudio del dominio afectivo. Esta engloba las creencias de los estudiantes sobre la utilidad de las matemáticas tanto en su día a día como en su futuro académico o profesional (Fennema \& Sherman, 1979). En este sentido, se define el concepto de utilidad: "la utilidad es un valor que hombres y mujeres exteriorizan en la resolución de problemas mediante creencias, sentimientos y capacidades desde sus contextos multiculturales".

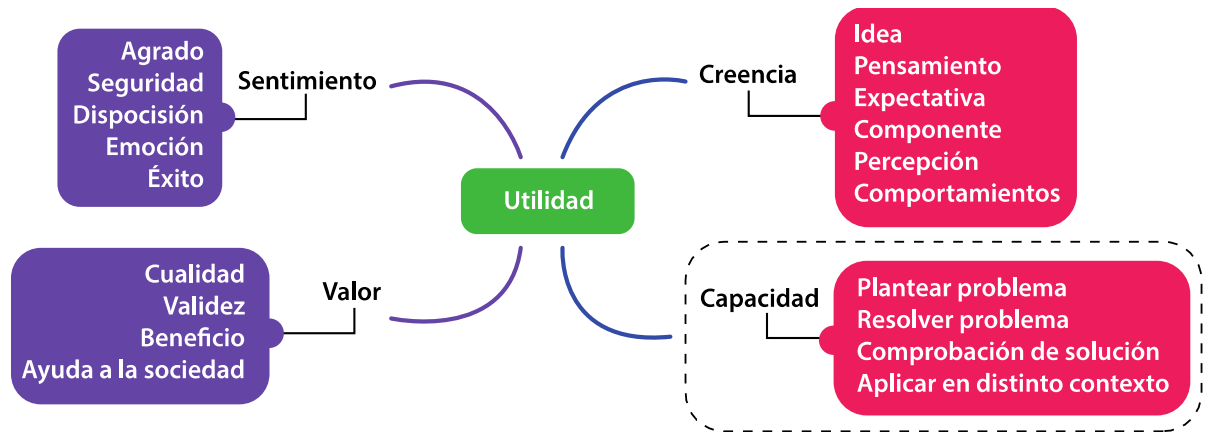

Figura 6: Concepción de utilidad hacia las matemáticas. 


\section{Conclusiones}

Esta investigación ha analizado los significados propios y compartidos sobre las actitudes hacia las matemáticas por estudiantes universitarios en contextos multiculturales. Los significados propios sobre actitudes hacia las matemáticas son cualidades de hombres y mujeres que se interiorizan en actuaciones, conductas, sentimientos, valores, capacidades y comportamientos hacia un constructo u objeto mediante el estímulo a una reacción o una petición en la resolución de problemas en contextos multiculturales.

Los significados compartidos sobre actitudes hacia las matemáticas son procesos participativos, democráticos e inclusivos que ponen de manifiesto la variabilidad declarada en las formas que puede interiorizar y exteriorizar la ansiedad, confianza, utilidad, motivación y agrado hacia las matemáticas los estudiantes universitarios. Además, acciones compartidas que permiten una articulación coherente al momento de realizar prácticas operativas y discursivas en la resolución de una práctica matemática. En este contexto destacamos:

1. La ansiedad son comportamientos de inseguridad, desconfianza y preocupación de hombres y mujeres que se exteriorizan como conductas, sentimientos y capacidades en el momento de sentir y pensar la solución de un problema en contexto multiculturales.

2. El agrado es un sentimiento de disfrute, felicidad y comportamientos que hombres y mujeres manifiestan al momento de usar su capacidad de resolver problemas en contextos multiculturales.

3. La motivación es el conjunto de habilidades, capacidades y comportamientos que hombres y mujeres interiorizan de forma interna y externa para auto estimularse su estado de ánimo y sentimientos de cara a la resolución de problemas en contextos multiculturales.

4. La confianza es un sentimiento de éxito y bienestar que provoca en hombres y mujeres el valor y la capacidad de construir ideas para resolver problemas a partir del reconocimiento y la familiarización del contexto multicultural.

5. La utilidad es un valor que hombres y mujeres exteriorizan en la resolución de problemas mediante creencias, sentimientos y capacidades desde sus contextos multiculturales.

En definitiva, resulta importante conocer los significados propios y compartidos sobre las actitudes porque constituyen una información valiosa para el diseño, planificación y evaluación de los procesos didácticos y pedagógicos por parte del profesorado, y así generar procesos de aprendizaje en el estudiantado, quienes van a seguir interactuando con las matemáticas en el ámbito académico, profesional y personal a lo largo de toda su vida.

\section{Agradecimiento}

Esta publicación obtuvo el financiamiento de: El Fondo de Asistencia Internacional de los Estudiantes y Académicos Noruegos (SAIH).

\section{Lista de referencias}

Allport, G. W. (1935). Attitudes. In C. Murchinson (Ed.) A Handbook of social psychology (798-844). Worcester: Clark University Press.

Auzmendi, E. (1992). Las actitudes hacia las matemática-estadística en las enseñanzas medias y universitarios. Bilbao: Mensajero.

Babbie, E. (1974). The practice of social research. Belmont. California: Wadsworth Publishing Company.

Bisquerra, R. (2012). Metodología de la Investigación Educativa. Madrid: Muralla. 
Bursal, M., \& Paznokas, L. (2006). Mathematics anxiety and pre-service elementary teachers' confidence to teach Mathematics and science. School Science and Mathematics, 106(4), 173-179. https://doi.org/10.1111/j.1949-8594.2006.tb18073.x

Cardoso, E., \& Vanegas E. (2012). Diagnóstico sobre la actitud hacia las matemáticas en tres posgrados en administración de empresa. Revista Electrónica Educard, 16(2), 237-253. https://doi.org/10.15359/ ree.16-2.15

Fennema, E., \& Sherman, J. (1976). Mathematics Attitudes Scales: Instruments designed to measure attitudes toward the learning of Mathematics by females and males. Journal for Research in Mathematics Education, 7(5), 324-326. https://doi.org/10.2307/748467

Flores, W. O., \& Auzmendi, E. (2015). Análisis de la estructura factorial de una escala de actitudes hacia las matemáticas. Aula de Encuentro, 17(1), 45-77. https://revistaselectronicas.ujaen.es/index.php/ADE/ article/view/2256

Flores, W. O., \& Auzmendi, E. (2018). Actitudes hacia las matemáticas en la enseñanza universitaria y su relación con las variables género y etnia. Profesorado. Revista de Currículum y Formación de Profesorado, 22(3), 231-251. https://doi.org/10.30827/profesorado.v22i3.80oo

Flores, W., O. (2019). Actitudes hacia las matemáticas en la resolución de problemas y su relación con la investigación propia. Managua: Editorial URACCAN.

Furner, J., \& Berman, B. (2003). Math anxiety: overcoming major obstacle to the improvement of student's math performance. Childhood Education. 1-5. https://doi.org/10.1080/00094056.2003.10522 220

Gadamer, H. G. (2000). Dialéctica de Hegel. Ediciones Cátedra.

Gómez-Chacón, I. (2000). Matemática Emocional: Los afectos en el aprendizaje Matemático. Madrid: Narcea.

Gresham, G. (2010). A study exploring excepcional education preservice teacher Mathematics anxiety. IUMPST: The Journal 4 (Curriculum).

Guerrero, E., Blanco, L., \& Vicente, F. (2002). Trastornos emocionales ante la educación matemática. Pirámide.

Heidegger, M. (1982). The Basic Problems of Phenomenology, trans. A. Hofstadter.

Krueger, R. (1991). El grupo de discusión. Guía práctica para la investigación aplicada. Madrid: Pirámide.

Likert, R. (1976). Una técnica para la medición de actitudes. Buenos Aires: Nueva Visión.

Mato, M. \& De la Torre, E. (2010). Evaluación de las actitudes hacia las matemáticas y el rendimiento académica. PNA, 5(1), 197-208.

Melich, J. C. (1994). Del extraño al cómplice: La educación en la vida cotidiana (Vol. 3). Barcelona: Anthropos Editorial.

Palacios, A., Arias, V., \& Arias, B. (2014). Las actitudes hacia las matemáticas: construcción y validación de un instrumento para su medida. Psicodidáctic, 19(1), 67-91. http://hdl.handle.net/11162/171139

Ricoeur, P. (200o). Teoría de la interpretación. Discurso y excedente de sentido. Editorial Siglo Veintiuno: Buenos Aires. 
Rokeach, M. (1968). A theory of organization and change with in value attitude system 1. Journal of socal issues, 24(1), 13-33. https://doi.org/10.1111/j.1540-456o.1968.tbo1466.x

Santrock, J. (2001). Psicología de la educación. Motivación y Aprendizaje. México: McGraw-Hill.

Wenger, E. C., \& Snyder, W. M. (2000). Communities of practice: The organizational frontier. Harvard Businees Review, 78(1), 139-145.

Wethrell, M., \& Potter, J. (1996). El análisis del discurso y la identidicación de los repertorios interpretativos. Recuperado en: http://gemma.atipic.net/pdf/326AD10405E.pdf

Wodak, R., \& Meyer, M. (2009). Critical discourse analysis: history, agenda, theory and methodology. In $R$. Wodak \& M. Meyer (Eds.). Methods of critical discourse analysis. London: SAGE. 